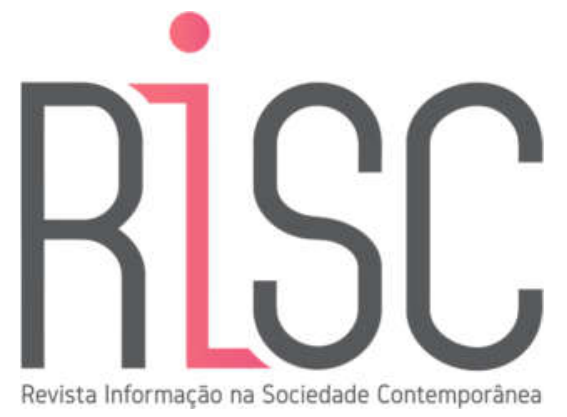

\title{
A Homogeneidade da Inclusão Digital no Brasil: Sonho ou Realidade?
}

\author{
Laleska Lebioda \\ Mestra em Administração \\ Universidade do Estado de Santa Catarina, Florianópolis, SC, Brasil; \\ https://orcid.org/0000-0002-0301-3873 E-mail: laleskalebioda@hotmail.com
}

Gabriela Ostrovski Cabral

Mestra em Administração

Universidade do Estado de Santa Catarina, Florianópolis, SC, Brasil;

https://orcid.org/0000-0003-1564-9753 E-mail: gabrielaostrovski@hotmail.com

\section{Rafael Tezza}

Doutor em Engenharia de Produção

Universidade do Estado de Santa Catarina, Florianópolis, SC, Brasil;

https://orcid.org/0000-0002-6539-4608 E-mail: rafael.tezza@udesc.br

Submetido em: 26-10-2019 Reapresentado em: 20-12-2019 Aceito em: 30-12-2019

\section{RESUMO}

A inclusão digital visa combater a exclusão digital, ou seja, diminuir a lacuna que existe tanto para oportunidade de acesso a tecnologias de informação e comunicação, quanto ao acesso à internet. A exclusão digital reflete as diferenças entre países e entre regiões de um mesmo país. Desta forma, o presente estudo tem como finalidade verificar se existe homogeneidade na inclusão digital entre as regiões do Brasil: Centro-Oeste, Nordeste, Norte, Sudeste e Sul levando em consideração o primeiro nível de um excluído digital, segundo Castells (1999), referente ao acesso as TICs. Para atingir o objetivo foi desenvolvida uma pesquisa de abordagem quantitativa descritiva, foram coletados dados secundários do Governo Federal 
do Brasil e do Centro Regional para o Desenvolvimento da Sociedade da Informação, no período de 2011-2014. Os resultados obtidos mostram que a diferença entre regiões no âmbito da desigualdade social também impacta no âmbito da inclusão digital, levando a conclusão de que não há homogeneidade da inclusão digital no Brasil.

Palavras-chave: inclusão digital; exclusão digital; desigualdade social

\section{The Homogeneity of Digital Inclusion in Brazil: Dream or Reality?}

\section{ABSTRACT}

Digital inclusion aims to combat the digital divide, that is, to close the gap that exists for both access to information and communication technologies and access to the Internet. The digital divide reflects the differences between countries and between regions within the same country. The present study aims to verify if there is a homogeneity in the digital inclusion between the regions in Brazil: Central West, Northeast, North, Southeast and South considering the first level of a digital excluded, according to Castells (1999), regarding access to ICTs. The study has a quantitative descriptive approach, secondary data were collected from the Federal Government of Brazil and from the Regional Center for the Development of the Information Society from 2011 to 2014. The results show that the difference between regions in the scope of social inequality also impacts on digital inclusion, leading to the conclusion that there is no homogeneity of digital inclusion in Brazil.

Keywords: digital inclusion; digital divide; social inequality. 


\section{INTRODUÇÃO}

A expressão inclusão digital, de forma simplista, refere-se ao combate da exclusão digital, que depende de cinco fatores (a existência de infraestruturas físicas de transmissão; o acesso a equipamentos e à conexão individual; a alfabetização digital; a capacitação intelectual e inserção social do usuário e; o desenvolvimento e uso de conteúdos específicos às necessidades dos diversos segmentos da população). Cada um deles impacta de maneira única a desigualdade social (SORJ, 2003).

Na literatura científica, a discussão em torno da exclusão digital aborda o avanço das Tecnologias da Informação e Comunicação - TICs. Esse assunto, na literatura americana sob o título de "digital divide", é um desafio a ser superado (WARSCHAUER, 2003).

De acordo com Grossi, Costa e Santos (2013) a desigualdade social é um dos principais fatores que favorecem a exclusão digital, sendo necessário novas atitudes para diminuir a desigualdade no Brasil. Abordando as temáticas desigualdade social e inclusão digital, Grossi, Costa e Santos (2013, p.71) realizaram um estudo com o objetivo de "verificar as ações que envolvem programas e projetos de inclusão digital que o governo brasileiro tem oferecido e que promovem não apenas a inclusão digital, mas também a inclusão social". Da mesma forma, Sorj (2003) em seu livro "brasil@povo.com", faz uma reflexão sobre exclusão digital e seu vínculo com a desigualdade social.

Ressalta-se que nas últimas décadas o Brasil obteve avanços significativos na área social, porém, ainda não foi possível alterar de forma relevante as desigualdades entre regiões do país (SENADO, 2015a). Embora seja alvo de políticas públicas há décadas, a desigualdade entre as regiões no Brasil é um problema persistente (SENADO, 2015).

Segundo Castells (1999) existem três níveis de um indivíduo ser um excluído digital, sendo eles: o primeiro nível está ligado ao não acesso à rede de computadores, no segundo nível o indivíduo possui acesso, porém com baixíssima aptidão técnica e, por fim, no terceiro nível o indivíduo pode estar conectado à rede, porém não possui

Rev. Inf. na Soc. Contemp., Natal, RN, v. 3, n. 1, 2019 
discernimento sobre o que acessar, o que procurar, não sabendo como utilizar os recursos disponíveis em benefício próprio.

Diante do exposto, o presente estudo tem como objetivo verificar se há homogeneidade na inclusão digital, na dimensão do acesso, entre as regiões do Brasil.

\section{A INCLUSÃO DIGITAL}

A inclusão digital se consolida como a ocasião em que indivíduos têm a possibilidade e a capacidade de utilizar os novos meios de comunicação eletrônica disponíveis, obtendo com esta utilização serviços, informações, conhecimentos e relações que impulsionam a entrada de variadas culturas nas redes (TRAMONTIN; BORGES, 2007). Ressalta-se que caso as TICs sejam associadas, exploradas e promovidas adequadamente, torna-se possível incitar o desenvolvimento cognitivo do usuário (WARSCHAUER, 2003) e facilitar a capacidade do mesmo.

Existem dois modelos de distribuição de acesso à novas tecnologias: um entendendo o acesso, como o acesso físico às TICs, chamado de modelo de dispositivos, e outro entendendo o acesso como um suprimento constante de algo que dependa de uma infraestrutura anterior, que geralmente possui taxas mensais, como a eletricidade, nomeado de modelo de distribuição por canais (WARSCHAUER, 2003).

A ideia de tornar real a inclusão digital fundamenta-se em quatro pressupostos expostos por Silveira (2001): 1) o reconhecimento de que a falta da inclusão digital expande a miséria, além de dificultar o desenvolvimento do indivíduo; 2) a aceitação de que o mercado não oferecerá oportunidades para a inclusão, na era da informação, de pessoas carentes de dinheiro e conhecimento; 3) a velocidade da inclusão é fator crucial, tanto para a sociedade adquirir a capacidade de gerar inovações, quanto para estar apta para as oportunidades de desenvolvimento no contexto da mundialização; 4) a aceitação de que todo e qualquer indivíduo tem direito à liberdade de expressão e o direito de se comunicar. Assim sendo, a inclusão digital trata-se de uma questão de cidadania, tendo em vista que atualmente o direito à comunicação é sinônimo do direito a comunicação mediada por um computador (SILVEIRA, 2001). 
A exclusão digital, ou seja, o não estar incluído digitalmente, reforça a desigualdade social. Para lidar com esta problemática se faz necessário uma nova atitude e um novo olhar para lutar pela diminuição da desigualdade no Brasil (GROSSI; COSTA; SANTOS, 2013).

\subsection{A INCLUSÃO DIGITAL NO BRASIL}

Os programas governamentais voltados para o desenvolvimento da inclusão digital possuem como foco principal as dimensões passivas do acesso à internet (SORJ, 2003). Esta visão é corroborada por Albertin (2005), quando expõe que a dimensão do acesso às TICs é a principal dentre as ações estratégicas governamentais de inclusão digital.

No Brasil, o que ocorre não é diferente, pois a inclusão digital no país sempre foi vista como a participação dos indivíduos no uso das TICs (CABRAL FILHO; CABRAL, 2013). A apropriação das Tecnologias Digitais de Informação e Comunicação - TDICs ocorre através de programas de inclusão digital, permitindo assim que o cidadão se perceba como parte do mundo tecnológico (GROSSI; COSTA; SANTOS, 2013). Como exemplo, o Governo brasileiro tem tentado superar as desigualdades digitais no Brasil, proporcionando às classes marginalizadas acesso físico à tecnologia (NEMER; GROSS; TRUE, 2013).

Apoiando a afirmação dos autores, a Lei Federal no 9.998, de 17 de agosto de 2000, que instituiu o Fundo de Universalização dos Serviços de Telecomunicações FUST, possui como objetivo "subsidiar serviços de telecomunicações para as camadas mais pobres da população, bem como para aquelas residentes em locais onde a exploração comercial desses serviços não é viável" (BRASIL, 2014).

A política adotada baseia-se na suposição de que as TICs são o motor mais importante da prosperidade da sociedade, permitindo o uso global de novas tecnologias de comunicação que acabará por levar a uma enorme transformação social e econômica (NEMER; GROSS; TRUE, 2013). 
Outra estratégia adotada pelo Governo brasileiro foi a criação do Projeto Cidadão Conectado - Computador para Todos, através do Decreto no 5.542, de 20 de setembro de 2005. Em articulação com a iniciativa privada, o projeto facilita a aquisição de computadores, possibilitando a população que não tem acesso ao computador, a obtenção de um equipamento de qualidade, além de permitir acesso à internet (BRASIL, 2005; BRASIL, 2016).

Percebe-se, a partir do exposto, que existem uma série de aspectos políticos e sociais atrelados na questão da inclusão digital, evidenciando a forte conexão existente entre o papel do Estado e os benefícios sociais da inclusão digital, a partir do avanço do acesso e da capacitação dos cidadãos junto às TICs (CERQUINHO et al., 2015). E o que se percebe quanto ao Brasil é que ainda faltam projetos ligados a capacitação para um melhor desenvolvimento da inclusão digital.

\subsection{A EXCLUSÃO DIGITAL}

A expressão inclusão digital refere-se ao combate da exclusão digital. De acordo com Sorj (2003), existem cinco fatores dos quais a exclusão digital é dependente. Cada um deles impacta de forma específica sobre a desigualdade social

1. A existência de infraestruturas físicas de transmissão;

2. O acesso a equipamentos e à conexão individual (computador, modem, linha de acesso);

3. A alfabetização digital;

4. A capacitação intelectual e inserção social do usuário;

5. O desenvolvimento e uso de conteúdos específicos às necessidades dos diversos segmentos da população.

Ressalta-se que as duas primeiras são dimensões passivas do acesso à internet, ou seja, estão relacionadas com a existência e ao acesso às conexões e infraestruturas, enquanto as outras três estão relacionadas com o potencial de apropriação, que possibilitam a utilização plena das tecnologias pelas pessoas. Essa distinção é essencial para o desenvolvimento de procedimentos contra a exclusão digital (SORJ, 2003). 
Segundo Castells (1999) existem três grandes formas de um indivíduo ser um excluído digital, sendo elas: não ter acesso à rede de computadores; ter acesso ao computador, porém com baixíssima aptidão técnica; estar conectado à rede, porém não ter discernimento sobre o que acessar, o que procurar, não sabendo como utilizar os recursos disponíveis em benefício próprio.

A partir dos conceitos apresentados por Castells (1999), Warschauer (2003), Sorj (2003), Cabral Filho e Cabral (2013), relacionados com o acesso as TICs e a conexão, este estudo analisou a inclusão digital sob a dimensão do acesso, sendo esta dimensão entendida pelo acesso e a existência de infraestruturas e conexões.

Destaca-se que o significado de exclusão social da Organisation for Economic Cooperation and Development - OECD foi fator motivador para a definição do objetivo deste trabalho. De acordo com a OECD (2001) o termo "exclusão digital" refere-se a lacuna que existe tanto para oportunidade de acesso a tecnologias de informação e comunicação, quanto ao acesso à internet. Essa lacuna está presente entre indivíduos, grupos, negócios e áreas geográficas em diferentes níveis socioeconômicos. A exclusão digital reflete as diferenças entre países e entre regiões de um mesmo país.

\section{MÉTODO}

A fim de atingir o objetivo desta pesquisa foi desenvolvido um estudo de abordagem quantitativa descritiva. Esta abordagem tem como objetivo a identificação, registro e análise de variáveis que se relacionam com o fenômeno estudado (PEROVANO, 2014) sem sofrer a manipulação pelo pesquisador (HEERDT; LEONEL, 2007). Conforme já mencionado, o fenômeno abordado neste estudo é a inclusão digital. Com relação aos procedimentos, foi realizado um levantamento de dados com corte transversal que visa a avaliação de uma determinada população ao longo de um período de tempo (GRAY, 2012). Este estudo abordou os anos de 2011, 2012, 2013 e 2014. 
Realizou-se a coleta de dados secundários ${ }^{1}$ do Governo, através do Portal Brasileiro de Dados Abertos e do Centro Regional para o Desenvolvimento da Sociedade da Informação - CETIC, por meio do site da instituição. Portanto, a pesquisa possui como seu universo a população brasileira que possuía acesso à internet e/ou possuía alguma TIC (televisão; antena parabólica; rádio; telefone fixo; telefone móvel) nos anos de 2011 a 2014.

Separou-se as variáveis em latente e observada, para então submete-las ao teste que determina estatisticamente se há diferença nas médias das amostras, para assim responder ao objetivo de verificar se há homogeneidade na inclusão digital, na dimensão do acesso, entre as regiões no Brasil.

Desta forma, o estudo apresenta, inicialmente, uma variável latente representada aqui por 16 variáveis observadas, conforme exposto na Tabela 1.

Tabela 1 - Descrição das variáveis latentes e observadas

\begin{tabular}{|c|c|}
\hline $\begin{array}{l}\text { Variável } \\
\text { Latente }\end{array}$ & Variável Observada \\
\hline \multirow{12}{*}{$\begin{array}{l}\text { Inclusão } \\
\text { Digital }\end{array}$} & VO.1 Proporção de assinantes de TV por assinatura \\
\hline & VO.2 Proporção de acessos móveis de internet banda larga \\
\hline & VO.3 Proporção de municípios cobertos por internet banda móvel \\
\hline & VO.4 Proporção de municípios já atendidos pelo $\mathrm{PNBL}^{1}$ \\
\hline & VO.5 Proporção de acessos de internet fixa em serviço \\
\hline & VO.6 Proporção de acessos telefônicos móveis \\
\hline & VO.7 Proporção de indivíduos que possuem televisão \\
\hline & VO.8 Proporção de indivíduos que possuem antena parabólica \\
\hline & VO.9 Proporção de indivíduos que possuem TV por assinatura \\
\hline & VO.10 Proporção de indivíduos que possuem rádio \\
\hline & VO.11 Proporção de indivíduos que possuem telefone fixo \\
\hline & VO.12 Proporção de indivíduos que possuem telefone móvel \\
\hline
\end{tabular}

\footnotetext{
${ }^{1}$ Série histórica de indicadores dos resultados governamentais relativos à Inclusão Digital. PGI é um repositório de informações estruturadas (indicadores) à feição dos processos de governança para o Governo Federal; ou seja, um repositório de compartilhamento de indicadores de políticas públicas. Segundo o repositório, os dados das séries de indicadores são fornecidos por seu valor histórico e não serão mais atualizados após dezembro/2014.
} 


\begin{tabular}{c|l}
\hline & VO.13 Proporção de indivíduos que possuem videogame/console \\
\cline { 2 - 2 } & VO.14 Proporção de indivíduos que possuem computador de mesa \\
\cline { 2 - 2 } & VO.15 Proporção de indivíduos que possuem computador portátil \\
\cline { 2 - 2 } & VO.16 Proporção de indivíduos que possuem tablet \\
\hline & PNBL - Programa Nacional de Banda Larga \\
Fonte: elaborado pelos autores (2019)
\end{tabular}

Primeiramente, realizou-se uma análise fatorial exploratória - AFE, por meio do software SPSS 21. O objetivo da utilização desta análise foi o de verificar se as 16 variáveis observadas estão medindo o mesmo traço latente, neste caso a inclusão digital, ou gerando um outro construto. Além disso, esta análise pode servir para eliminação de itens não alinhados estatisticamente com o objeto de estudo. Nesta etapa, algumas estatísticas precisam ser observadas para verificar a adequação dos dados, como o KMO (Medida Kaiser-Meyer-Olkin)², as cargas fatoriais e comunalidade. É comum, segundo Hair et al. (2005) utilizar-se KMO >0,5, cargas fatoriais e comunalidade das variáveis observadas também maiores que 0,5.

Verificou-se a normalidade dos dados através da aplicação do teste Shapiro-Wilk e aplicou-se o teste Kruskal Wallis ${ }^{3}$ para responder ao objetivo da pesquisa, verificar se há homogeneidade na inclusão digital, na dimensão do acesso, entre as regiões no Brasil: Centro-Oeste, Nordeste, Norte, Sudeste e Sul. Os resultados serão apresentados na próxima seção.

\section{RESULTADOS E DISCUSSÕES}

Nesta parte serão apresentados os resultados obtidos através dos métodos expostos acima. O primeiro resultado obtido através da análise fatorial exploratória mostra que

\footnotetext{
${ }^{2}$ A estatística Kaiser-Meyer-Olkin (KMO) "compara as correlações simples com as correlações parciais [...]; avalia a adequação da amostra quanto ao grau de correlação parcial entre as variáveis" (FÁVERO et al., 2009, p. 241).

${ }^{3}$ O teste Kruskal Wallis é um teste não paramétrico (não assume distribuição normal) que compara amostras independentes, identificando se existe diferença de médicas entre as amostras. Contudo, não indica entre quais amostras há diferença, indicando apenas que ao menos uma amostra possui diferença entre as demais (MCKIGHT; NAJAB, 2010).
} 
a Medida Kaiser-Meyer-Olkin (KMO) é igual a 0,599. Segundo Hair et al. (1987), esta medida deve estar entre 0,5 e 1,0 para ser aceita. Fávero et al. (2009, p. 241) complementam que "o valor de KMO próximo de 0 indica que a análise fatorial pode não ser adequada, pois existe uma correlação fraca entre as variáveis. Por outro lado, quanto mais próximo de 1 o seu valor, mais adequada é a utilização da técnica". Sendo assim, o resultado mostra-se satisfatório.

Seguindo com a análise fatorial exploratória, observou-se as cargas fatoriais e o número de dimensões indicado, neste caso com base nos autovalores. Os resultados mostram que o primeiro autovalor é responsável por $57 \%$ da variância total, sustentando a unidimensionalidade dos dados. A tabela 2 apresenta as cargas fatoriais e a comunalidade de cada item.

\section{Tabela 2 - Cargas fatoriais e comunalidade}

\begin{tabular}{|c|c|c|}
\hline Variável & Carga & Comunalidade \\
\hline VO.1 & 0,961 & ,923 \\
\hline VO. 2 & 0,955 & ,912 \\
\hline VO.3 & 0,875 & ,765 \\
\hline VO.4 & 0,939 & ,881 \\
\hline V0.5 & 0,758 & ,575 \\
\hline V0.6 & 0,746 & ,557 \\
\hline V0.7 & 0,786 & ,618 \\
\hline VO.8 & 0,767 & ,589 \\
\hline VO.9 & 0,965 & 932 \\
\hline VO.10 & 0,832 & ,693 \\
\hline V0.11 & 0,993 & 986 \\
\hline V0.12 & 0,861 & ,742 \\
\hline VO.13 & 0,959 & ,919 \\
\hline VO.14 & 0,955 & ,912 \\
\hline V0.15 & 0,894 & 800 \\
\hline V0.16 & 0,905 & 819 \\
\hline
\end{tabular}

Fonte: Elaborado pelos autores (2019).

As comunalidades representam a proporção da variância para cada variável incluída na análise que é explicada pelos componentes extraídos. Caso alguma variável possuir sua comunalidade com valor abaixo do esperado a variável deve ser excluída e a análise fatorial deve ser realizada novamente. Observa-se na Tabela 2 que todas as variáveis apresentaram uma carga fatorial maior do que 0,5 na mesma dimensão, 
indicando de estão medindo o mesmo traço latente e nenhum item precisou ser eliminado.

Desta forma, o estudo teve sua continuidade não excluindo nenhuma das variáveis, obtendo-se as seguintes hipóteses para serem testadas através do constructo inclusão digital (Quadro 1).

\section{Quadro 1 - Hipóteses de pesquisa}

\begin{tabular}{|l|l|}
\hline Hipótese & \\
\hline H1 & Há diferença na proporção de assinantes de TV por assinatura \\
\hline H2 & Há diferença na proporção de acessos móveis de internet banda larga \\
\hline H3 & Há diferença na proporção de municípios cobertos por internet banda móvel \\
\hline H4 & Há diferença na proporção de municípios já atendidos pelo PNBL \\
\hline H5 & Há diferença na proporção de acessos de internet fixa em serviço \\
\hline H6 & Há diferença na proporção de acessos telefônicos móveis \\
\hline H7 & Há diferença na proporção de indivíduos que possuem televisão \\
\hline H8 & Há diferença na proporção de indivíduos que possuem antena parabólica \\
\hline H9 & Há diferença na proporção de indivíduos que possuem TV por assinatura \\
\hline H10 & Há diferença na proporção de indivíduos que possuem rádio \\
\hline H11 & Há diferença na proporção de indivíduos que possuem telefone fixo \\
\hline H12 & Há diferença na proporção de indivíduos que possuem telefone móvel \\
\hline H13 & Há diferença na proporção de indivíduos que possuem videogame/console \\
\hline H14 & Há diferença na proporção de indivíduos que possuem computador de mesa \\
\hline H15 & Há diferença na proporção de indivíduos que possuem computador portátil \\
\hline H16 & Há diferença na proporção de indivíduos que possuem tablet \\
\hline & \\
\hline
\end{tabular}

Fonte: Elaborado pelos autores (2019)

Após a elaboração das hipóteses, realizou-se a verificação da normalidade dos dados através da aplicação do teste Shapiro-Wilk, conforme apresenta a Tabela 3. Numa amostra normal a distribuição de dados ocorre de forma simétrica. Populações com distribuição normal são difíceis de se obter, o que acontece frequentemente é uma distribuição aproximadamente normal. A tabela 3 mostra os valores de sig ( $p$-Values) ${ }^{4}$, os quais devem ser maiores que 0,05 para serem considerados normais. Cinco das

\footnotetext{
${ }^{4}$ Os $p$-Values indicam o quão compatíveis os dados são com um modelo estatístico especificado, fornecendo uma abordagem para resumir a compatibilidade entre um conjunto específico de dados e um modelo proposto para os dados (WASSERSTEIN et al., 2016).
} 
dezesseis hipóteses foram consideradas sem normalidade, sendo elas: H3, H5, H12, H14

e H16. A normalidade ou não dos dados determinou o tipo de teste para suportar ou refutar as hipóteses formuladas.

Tabela 3 - Teste de normalidade Shapiro-Wilk
\begin{tabular}{c|c}
\hline Hipótese & Sig \\
\hline $\mathrm{H} 1$ &, 086 \\
\hline $\mathrm{H} 2$ &, 118 \\
\hline $\mathrm{H} 3$ &, 035 \\
\hline $\mathrm{H} 4$ &, 179 \\
\hline $\mathrm{H} 5$ &, 000 \\
\hline $\mathrm{H} 6$ &, 449 \\
\hline $\mathrm{H} 7$ &, 100 \\
\hline $\mathrm{H} 8$ &, 585 \\
\hline $\mathrm{H} 9$ &, 490 \\
\hline $\mathrm{H} 10$ &, 180 \\
\hline $\mathrm{H} 11$ &, 053 \\
\hline $\mathrm{H} 12$ &, 039 \\
\hline $\mathrm{H} 13$ &, 240 \\
\hline $\mathrm{H} 14$ &, 044 \\
\hline $\mathrm{H} 15$ &, 471 \\
\hline $\mathrm{H} 16$ &, 003 \\
\hline Fonte: Elaborado pelos autores (2019)
\end{tabular}

À vista disso, com o propósito de responder ao objetivo da pesquisa, utilizou-se o teste não-paramétrico (amostras sem normalidade) Kruskal-Wallis (Tabela 4).

Tabela 4 - Teste Kruskal Wallis

\begin{tabular}{l|l|l}
\hline \multicolumn{1}{c|}{ Hipótese } & Sig & \multicolumn{1}{|c}{ Resultado } \\
\hline H1 - Há diferença na proporção de assinantes de TV por assinatura &, 002 & Suportada \\
\hline H2 - Há diferença na proporção de acessos móveis de internet banda larga &, 549 & Refutada \\
\hline H3 - Há diferença na proporção de municípios cobertos por internet banda móvel &, 312 & Refutada \\
\hline H4 - Há diferença na proporção de municípios já atendidos pelo PNBL &, 461 & Refutada \\
\hline H5 - Há diferença na proporção de acessos de internet fixa em serviço &, 003 & Suportada \\
\hline H6 - Há diferença na proporção de acessos telefônicos móveis &, 046 & Suportada \\
\hline H7 - Há diferença na proporção de indivíduos que possuem televisão &, 011 & Suportada \\
\hline H8 - Há diferença na proporção de indivíduos que possuem antena parabólica &, 019 & Suportada \\
\hline H9 - Há diferença na proporção de indivíduos que possuem TV por assinatura &, 004 & Suportada \\
\hline H10 - Há diferença na proporção de indivíduos que possuem rádio &, 002 & Suportada \\
\hline H11 - Há diferença na proporção de indivíduos que possuem telefone fixo &, 001 & Suportada \\
\hline H12 - Há diferença na proporção de indivíduos que possuem telefone móvel &, 043 & Suportada \\
\hline H13 - Há diferença na proporção de indivíduos que possuem videogame/console &, 001 & Suportada \\
\hline
\end{tabular}




\begin{tabular}{l|l|l}
\hline H14 - Há diferença na proporção de indivíduos que possuem computador de mesa &, 002 & Suportada \\
\hline H15 - Há diferença na proporção de indivíduos que possuem computador portátil &, 017 & Suportada \\
\hline H16 - Há diferença na proporção de indivíduos que possuem tablet &, 978 & Refutada \\
\hline
\end{tabular}

Fonte: Elaborado pelos autores (2019)

Os resultados apresentados na Tabela 4 mostram que das dezesseis hipóteses testadas, doze foram suportadas $(\mathrm{Sig}<0,05)$, ou seja, doze hipóteses apresentam diferença na inclusão digital, de uma região para outra do Brasil e apenas quatro hipóteses foram refutadas, $\mathrm{H} 2, \mathrm{H} 3, \mathrm{H} 4$ e H16 mostrando que o acesso móvel no Brasil, vem de certa forma reduzindo, mesmo que em pequena escala, as diferenças na inclusão digital. Percebe-se isso observando que as hipóteses refutadas ao nível de 0,05 (H2 e H3), estão relacionadas ao acesso e a cobertura móvel. Além disso, caso fosse considerado nível de significância de 0,01 nas hipóteses H6 e H12 também seriam refutadas, as quais tratam respectivamente de proporção de acessos telefônicos móveis e proporção de indivíduos que possuem telefone móvel. Isto deve-se, particularmente a popularização dos dispositivos móveis (celulares e tablets) no Brasil, bem mais do que acesso a computadores de mesa, TV por assinatura, banda larga, radio, telefone fixo, etc.

Segundo Pellanda (2009), isso se deve ao fato de áreas populosas, mas informais, como as favelas ou áreas rurais, não terem infraestrutura para as ligações de telefone fixo e TV por assinatura, aliado ao desinteresse econômico das empresas. Entretanto o autor argumenta que a tecnologia sem fio transpõe este problema por não precisar de ligações diretas com as residências. Essa flexibilidade, aliada a uma expansibilidade, é um dos principais fatores de inclusão digital da tecnologia móvel.

Grossi, Costa e Santos (2013), afirmam que dentre os fatores que favorecem a exclusão digital, a desigualdade social ocupa um dos primeiros lugares, o que é refletido em bens como televisão, antena parabólica, computadores, acesso a TV por assinatura entre outros, como foi observado nos resultados do presente estudo. Fato este reduzido com as iniciativas públicas e privadas de expansão móvel. Tal fenômeno, ocorre em partes, segundo Pellanda (2009) dado que o Brasil possui características similares à África, onde a falta de telefones fixos também obrigou os países a pularem direto para a tecnologia móvel. 
Ressalta-se que, através do teste Kruskal Wallis foi possível identificar a existência de diferença da inclusão digital, na dimensão do acesso, entre as regiões do Brasil, contudo, não se identificou entre quais regiões há diferença. Os resultados indicam que ao menos uma região possui diferença entre as demais, mostrando que não há homogeneidade da inclusão digital no Brasil.

\section{CONCLUSÃO}

O objetivo desta pesquisa foi verificar se há homogeneidade na inclusão digital entre as regiões no Brasil: Centro-Oeste, Nordeste, Norte, Sudeste e Sul levando em consideração o primeiro nível de um excluído digital, segundo Castells (1999), referente ao acesso as TICS. Analisando os resultados verifica-se que a homogeneidade ainda é um sonho a ser alcançado.

Quando lidamos com a ideia de homogeneidade no processo de inclusão digital de uma população esbarramos na dimensão social e suas desigualdades (GROSSI; COSTA; SANTOS, 2013) e em um país como o Brasil, com suas dimensões continentais e a natureza distinta de suas regiões estes aspectos evidenciam-se. Portanto, o resultado da pesquisa já era imaginado pelos autores, sendo confirmado pelos testes realizados, os quais mostraram que apenas quatro variáveis analisadas apresentaram homogeneidade, evidenciando a predominância de heterogeneidade sobre as dimensões de acesso da inclusão digital entre as regiões brasileiras.

$O$ ineditismo deste estudo centra-se em ser um dos primeiros esforços empíricos no Brasil para analisar a que ponto está a inclusão digital no país. Além disso, os resultados do estudo servirão de base para pesquisas futuras mais abrangentes, envolvendo as demais dimensões de análise.

Os resultados encontrados auxiliam o Governo a tomar iniciativas que realmente combatam a exclusão social, tanto no âmbito do acesso, quanto a projetos mais elaborados e que envolvam também a capacitação dos usuários, bem como para empresas que trabalham em atividades específicas relacionadas ao constructo, para a elaboração de projetos futuros e estudos de mercado em TICs. 
Sugere-se como pesquisas futuras estudos que tenham como abordagem: conteúdo e linguagem, alfabetização e educação, além de estruturas comunitárias e institucionais (WARSCHAUER, 2003), pois, mesmo tendo atingido o objetivo proposto, o estudo apresenta algumas limitações. A primeira limitação refere-se ao tipo de corte utilizado para a operacionalização da pesquisa, observando que apenas foi envolvida a dimensão do acesso da inclusão digital, sendo esta a dimensão mais considerada nas ações estratégicas governamentais de inclusão digital (ALBERTIN, 2005).

Ainda no âmbito de acesso, a variável smartphone não foi analisada separadamente, ela foi abordada juntamente com a variável telefone móvel, pois o banco de dados utilizado não faz distinções entre os tipos de aparelhos. Entende-se que seria interessante abordar o smartphone separadamente, devido ao aumento do uso desta tecnologia. Segundo a 27ạ Pesquisa Anual de Administração e Uso de Tecnologia de Informação nas Empresas (MEIRELLES, 2016) o número de smartphones em uso no Brasil chegará em 2018 a 236 milhões, hoje já existem mais smartphones do que computadores e a projeção de densidade de dispositivos conectados à internet será de dois por habitante.

\section{Agradecimentos}

Agradecimento à fundação CAPES - Coordenação de Aperfeiçoamento de Pessoal de Nível Superior

\section{REFERÊNCIAS}

ALBERTIN, Alberto Luiz. A Tecnologia de Informação e o Indivíduo: Propondo um Modelo de Adoção de Tecnologia para a Inclusão Digital. São Paulo: FGV/EASP GVPESQUISA, 2005. Disponível em: https://pesquisaeaesp.fgv.br/publicacoes/gvp/tecnologia-de-informacao-e-o-individuo-propondo-ummodelo-de-adocao-de-tecnologia. Acesso em: julho de 2019.

BRASIL. Decreto no 5.542, de 20 de setembro de 2005. Institui o Projeto Cidadão Conectado - Computador para Todos, no âmbito do Programa de Inclusão Digital, e dá outras providências. Disponível em: http://www.planalto.gov.br/ccivil 03/ ato20042006/2005/decreto/D5542.htm. Acesso em: julho de 2019. 
BRASIL. Governo Eletrônico. Projeto Cidadão Conectado - Computador para todos. Abril de 2016. Disponível em: http://www.governoeletronico.gov.br/eixos-deatuacao/cidadao/inclusao-digital/projeto-cidadao-conectado-computador-para-todos. Acesso em: julho de 2019.

BRASIL. Ministérios das Comunicações. FUST. Maio de 2014.

CABRAL FILHO, Adilson. Vaz.; CABRAL, Eula. Dsantas. Taveira. Mapeamento da inclusão digital no Brasil. Revista Eptic Online. São Carlos, v.15, n.1, p. 4-19, 2013. Disponível em: http://www.seer.ufs.br/index.php/eptic/article/view/682. Acesso em: julho de 2019.

CASTELLS, Manuel. A sociedade em rede. São Paulo: Paz e Terra, 1999. v.1

CENTRO REGIONAL PARA O DESENVOLVIMENTO DA SOCIEDADE DA INFORMAÇÃO. Cetic.br. Cetic.br. Disponivel em: http://data.cetic.br/cetic/explore. Acesso em: julho de 2019.

CERQUINHO, Kleomara Gomes et al. Inclusão Digital para quê e para quem? Observação do Portal Inclusão Digital do Governo Federal Brasileiro. Cadernos Gestão Pública e Cidadania. São Paulo, v. 20, n. 67, p. 172-193, jul./dez., 2015. Disponível em: http://dx.doi.org/10.12660/cgpc.v20n67.32927. Acesso em: julho de 2019.

FÁVERO, Luiz Paulo et al. Análise de dados: modelagem multivariada para tomada de decisões Rio de Janeiro: Elsevier, 2009.

GOVERNO FEDERAL BRASILEIRO. Portal Brasileiro de Dados Abertos. Portal Brasileiro de Dados Abertos. Disponivel em: http://dados.gov.br/dataset/inclusao-digital. Acesso em: julho de 2019.

GRAY, David. E. Pesquisa no Mundo Real. 2. ed. Porto Alegre: Penso, 2012.

GROSSI, Márcia Gorett Ribeiro; COSTA, José Wilson da; SANTOS, Ademir José dos. A exclusão digital: o reflexo da desigualdade social no Brasil. Nuances: estudos sobre Educação, Presidente Prudente, v. 24, n. 2, p. 68-85, maio/ago., 2013. Disponível em: http://dx.doi.org/10.14572/nuances.v24i2.2480. Acesso em: julho de 2019.

HAIR, Joseph F. et al. Multivariate data analysis with readings. 3nd ed. New York: Macmillan, 1987.

HAIR, Joseph F. et al. Análise Multivariada de Dados. 5. ed. Porto Alegre: Bookman, 2005. 
HEERDT, Maur Luiz.; LEONEL, Vilson. Metodologia Científica e da Pesquisa. 5. ed. Palhoça: UnisulVirtual, 2007.

LÉON, Daniela Andrea Droguett. Análise Fatorial Confirmatória através dos Softwares R e Mplus. Monografia (Bacharelado em Estatística). Instituto de Matemática, Universidade Federal do Rio Grande do Sul, Porto Alegre, 2011. Disponível em: http://www.lume.ufrgs.br/bitstream/handle/10183/31630/000784196.pdf. Acesso em: julho de 2019.

MCKIGHT, Patrick E.; NAJAB, Julius. Kruskal-wallis test. The corsini encyclopedia of psychology, p. 1-1, 2010. Disponível em:

https://doi.org/10.1002/9780470479216.corpsy0491. Acesso em: dezembro de 2019.

MEIRELLES, Fernando S. Pesquisa Anual do Uso de TI nas Empresas, GVcia, FGVEAESP, 27a edição, 2016.

NEMER, David; GROSS, Shad; TRUE, Nic. Materializing digital inequalities: the digital artifacts of the marginalized in Brazil. Proceedings of the sixth international conference on information and communications technologies and development notes - ICTD '13. New York: ACM Press. v. 02, p. 108-111, 2013. Disponível em: http://dl.acm.org/citation.cfm?id=2517899.2517915. Acesso em: julho de 2019.

OECD. Understanding the Digital Divide. OECD Digital Economy Papers, No. 49, OECD Publishing. France, 2011. Disponível em: http://dx.doi.org/10.1787/236405667766. Acesso em: julho de 2019.

PEROVANO, Dalton Gean. Manual de Metodologia Científica para a Segurança Pública e Defesa Social. 1. ed. Curitiba: Jurua Editora, 2014.

SENADO FEDRAL. Secretaria de Comunicação Federal. Agenda social avançou, mas desigualdades persistem. Revista em discussão!, Brasília, Ano 6, no 26, p. 37 - 40. Set/ 2015a. Disponível em: https://www12.senado.leg.br/emdiscussao/edicoes/pactofederativo/realidade-brasileira/agenda-social-avancou-mas-desigualdades-persistem. Acesso em: julho de 2019.

SENADO FEDRAL. Secretaria de Comunicação Federal. Desigualdades ainda resistem às mudanças. Revista em discussão!, Brasília, Ano 6, no 26, p. 48 - 40. Set/ 2015b.

Disponível em: https://www12.senado.leg.br/emdiscussao/edicoes/pactofederativo/partilha-dos-tributos/desigualdades-ainda-resistem-as-mudancas. Acesso em: julho de 2019.

SILVEIRA, Sérgio Amadeu. Exclusão digital: a miséria na era da informação. São Paulo: Fundação Perseu Abramo, 2001. 
SORJ, Bernardo. Brasil@povo.com: a luta contra a desigualdade na Sociedade da Informação. Rio de Janeiro: Jorge Zahar Ed. Brasília: Unesco, 2003. Disponível em: http://www.bernardosorj.com/pdf/Brasil @ povo com.pdf. Acesso em: julho de 2019.

TRAMONTIN, Adriane; BORGES, Djalma Freire. Um caminho para a inclusão digital: o programa GESAC. Organizações \& Sociedade, Salvador, v. 14, n. 42, p. 167-184, set. 2007. Disponível em: http://dx.doi.org/10.1590/S1984-92302007000300010. Acesso em: julho de 2019.

WARSCHAUER, Mark. Technology and social inclusion: rethinking the digital divide. Massachusetts: MIT Press, 2003.

WASSERSTEIN, Ronald L. et al. The ASA's statement on p-values: context, process, and purpose. The American Statistician, v. 70, n. 2, p. 129-133, 2016. Disponível em: https://doi.org/10.1080/00031305.2016.1154108. Acesso em: dezembro de 2019.

\section{Contribuição dos autores}

Todos os autores colaboraram de igual maneira na concepção e elaboração do manuscrito. Todos os autores revisaram e aprovaram a versão final. 